\title{
A Rare Case of Thymic Gangliocytic Paraganglioma
}

\author{
Jung Wook Yang · Joungho Han · Hyun Woo Lee · Soo Youn Cho · Hong Kwan Kim ${ }^{1}$ \\ Departments of Pathology and ${ }^{1}$ Thoracic Surgery, Samsung Medical Center, Sungkyunkwan University School of Medicine, Seoul, Korea
}

Gangliocytic paraganglioma (GP) is a rare neuroendocrine tumor characterized by epithelioid endocrine cells, spindled schwann-like cells, and ganglion cell-like elements. ${ }^{1}$ These tumors almost always arise in the duodenum, particularly in the periampullary region. Extra-duodenal GPs are extremely unusu$\mathrm{al}^{2}{ }^{2}$ and we could not find any case of thymic GP in the English literature. Here, we report a case of thymic GP and describe its histological and immunohistochemical characteristics.

\section{CASE REPORT}

A 1.6-cm-sized anterior mediastinal mass was incidentally found in a 55-year-old female on computed tomography (CT) during work-up for thyroid carcinoma (Fig. 1A). During 12 months of follow-up, CT images showed no interval change. Partial thymectomy was performed, and the cut surface showed an ovoid, well-circumscribed, gray-tan mass measuring $2 \times 1.3$ $\mathrm{cm}$. Microscopically, the mass had an expanding border surrounded by normal thymic tissue (Fig. 1B). It was composed of spindle cells, ganglion-like cells, epithelioid cells, and fibrillary cells (Fig. 1C), which were haphazardly intermixed in most areas. The spindle cells were arranged in an intersecting fascicular pattern. The scattered ganglion-like cells showed large round nuclei with prominent nucleoli and a large amount of eosinophilic cytoplasm. The epithelioid cells had medium-sized round nuclei with indistinct nucleoli and a small or moderate amount of eosinophilic cytoplasm; they formed vague nests in some areas (Fig. 1D). The fibrillary cells also formed a vague or confluent

\section{Corresponding Author}

Joungho Han, MD

Department of Pathology, Samsung Medical Center, Sungkyunkwan University School of Medicine, 81 Irwon-ro, Gangnam-gu, Seoul 06351, Korea

Tel: +82-2-3410-2800, Fax: +82-2-3410-0025, E-mail: hanjho@skku.edu

Received: June 18, 2015 Revised: July 14, 2015

Accepted: July 14, 2015 nesting pattern in some areas. These cells had small round to ovoid dark nuclei with a fine chromatin pattern and fibrillary cytoplasm. Benign epithelial cells were also observed in approximately half of the cut surface area (Fig. 1F). These cells were intermixed with other components and showed a branching pattern (Fig. 1E). In some areas, they were located between nests of the fibrillary cells. There were several compressed cords and ducts formed by the epithelial cells. No cellular components showed atypia, and no mitosis was seen. On immunohistochemical stains (IHC) (Fig. 1G-K), spindle cells were positive for CD56 (1:200, Leica, Newcastle upon Tyne, UK), synaptophysin (1:50, Thermo Scientific, Rockford, IL, USA), and S-100 (1:5,000, Dako, Glostrup, Denmark). S-100 staining showed that some spindle cells were located adjacent to and in the nests of fibrillary cells. Ganglion-like cells were positive for CD56 and synaptophysin but negative for S-100 and AE1/AE3 (1:500, Dako). The epithelioid and fibrillary cells had the same results on IHC for CD56, synaptophysin, S-100, and AE1/AE3 as did the ganglion-like cells. However, the epithelial cells were only positive for $\mathrm{AE} 1 / \mathrm{AE} 3$ with a reticular or race-like cytoplasmic staining pattern (Fig. 1J). Some of the fibrillary and epithelioid cells were positive (Fig. 1K) for progesterone receptor (PR; 1:800, Leica), but most of them were negative (Fig. 1L).

\section{DISCUSSION}

The duodenum is the most common site of $\mathrm{GP}^{2}$ Only one case of GP in the posterior mediastinum has been reported, ${ }^{3}$ and we could not find any English literature cases of GP involving the thymus. We hypothesize that the rarity of thymic GP is due to minimal ganglioneuronal component in the thymus, as GP seems to be related to the amount of ganglioneuronal content in the involved organ. According to the World Health Organization classification, GP is a distinctive triphasic tumor 
consisting of haphazardly distributed epithelioid endocrine cells, spindled schwann-like cells, and ganglion cell-like elements. ${ }^{1}$ Composite paragangliomas with a ganglioneuromatous compo- nent also show the above three elements; ${ }^{4}$ however, the paraganglioma and ganglioneuroma components of the tumor are usually minimally admixed with an abrupt transition. The present
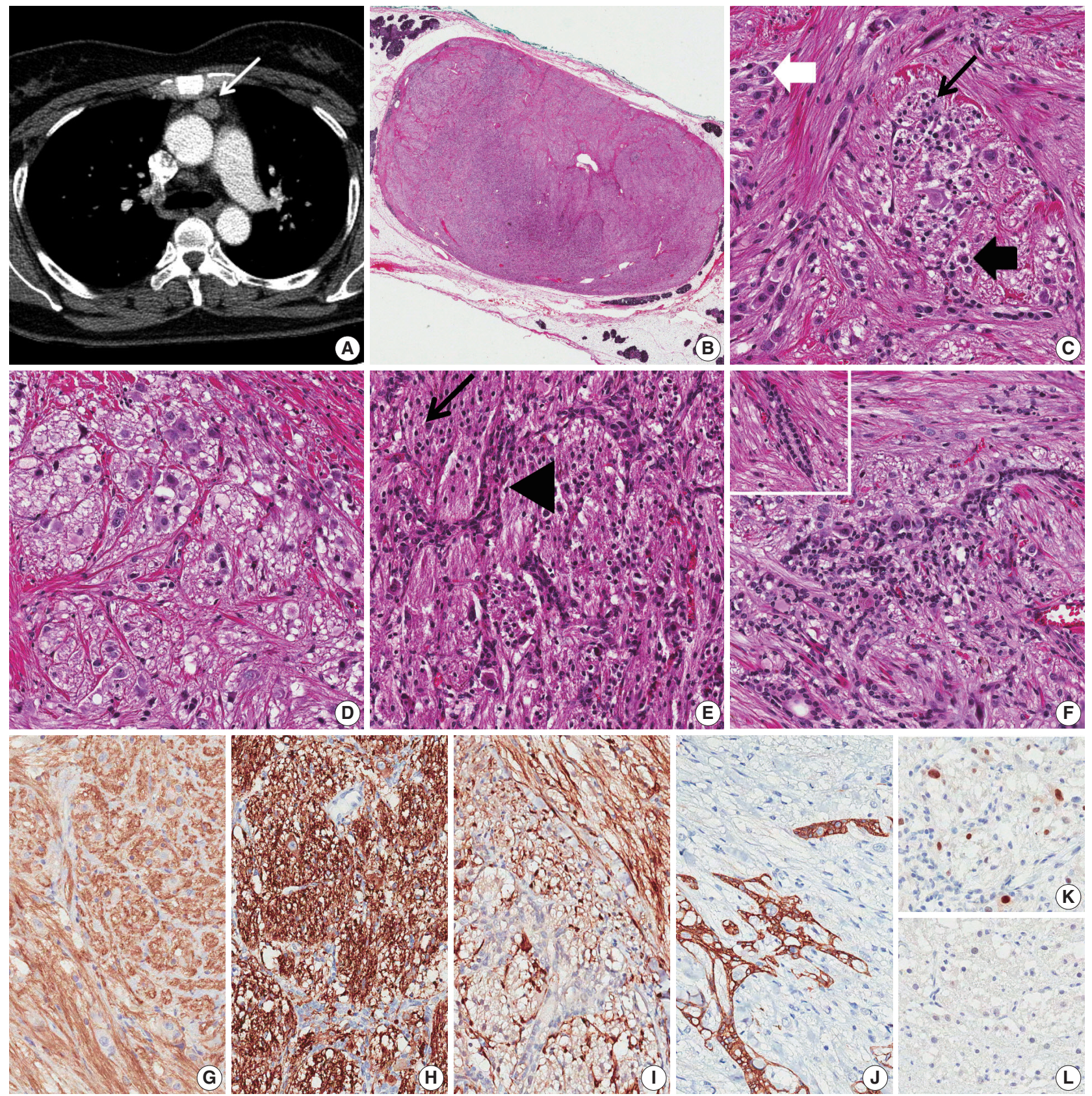

Fig. 1. Thymic gangliocytic paraganglioma. (A) Chest computed tomography reveals a well-circumscribed ovoid mass (white arrow) in the anterior mediastinum. (B) On low-power view, the mass shows an expanding border surrounded by normal thymic tissue. (C) Spindle cells show an intersecting fascicular pattern, and fibrillary cells show a vague or confluent nesting pattern (black arrow). Scattered ganglion-like cells (white thick arrow) and epithelioid cells (black thick arrow) are also noted. (D) Epithelioid cells form a vague nesting pattern. (E) Epithelial cells show a branching pattern (arrowhead) between the nests of fibrillary cells (black arrow). (F) In focal areas, the epithelial cells form compressed cords and ducts (left upper corner). (G, H) Staining with CD56 $(G)$ and synaptophysin $(H)$ is positive for spindle cells, ganglion-like cells, epithelioid cells, and fibrillary cells. (I) Only spindle cells are positive for S-100. Spindle cells are located adjacent to and within the nests of fibrillary cells. (J) On staining for AE1/AE3, the epithelial cells show a reticular or race-like cytoplasmic pattern. (K, L) Some of the fibrillary cells and epithelioid cells are positive $(K)$ for progesterone receptor, but most of them are negative $(L)$. 
case is consistent with GP, but there are two unusual components: fibrillary cells and epithelial cells. The fibrillary cells appeared similar to neuronal cells at first glance. Though the fibrillary cells showed a vague nesting pattern similar to carcinoids in some areas, the cells did not express AE1/AE3. Weissferdt $e t$ $a l^{5}$ reported that all of the studied thymic carcinoid tumors were positive for pancytokeratin. Based on these results, the tumors seem to be composed of neuronal-like cells rather than carcinoid-like cells. Okubo et al. ${ }^{6}$ reported that epithelioid cells in almost all cases of duodenal GP were positive for PR. In the present case, some fibrillary and epithelioid cells expressed PR. The fibrillary cells were most likely derived from the same progenitor cell as the epithelioid cells.

The epithelial cells might be entrapped thymic epithelial cells. The staining pattern for AE1/AE3 resembles that of thymic epithelial cells; however, the tumor had an expanding border, and the epithelial component was too extensive to be composed of entrapped thymic cells. Furthermore, the cells formed ducts in focal areas. Thus, the epithelial cells were probably one of the tumor components. Ogata et al. ${ }^{7}$ reported a case of duodenal GP with regional lymph node metastasis and a glandular component. The duodenal GP had small amounts of distinct glandular component with mucin. The metastatic tumor deposit in the dissected lymph nodes more prevalently exhibited glandular components with mucus compared to the primary site.

In conclusion, this rare case of thymic GP with neuronal-like and epithelial components shows an extremely unusual site of involvement and unusual tumor components.

\section{Conflicts of Interest}

No potential conflict of interest relevant to this article was reported.

\section{REFERENCES}

1. DeLellis RA, Lloyd RV, Heitz PU, Eng C. World Health Organization classification of tumors of pathology and genetics of tumors of endocrine organs. Lyon: IARC Press, 2004; 162-3.

2. Okubo Y, Wakayama M, Nemoto T, et al. Literature survey on epidemiology and pathology of gangliocytic paraganglioma. BMC Cancer 2011; 11: 187.

3. Weinrach DM, Wang KL, Blum MG, Yeldandi AV, Laskin WB. Multifocal presentation of gangliocytic paraganglioma in the mediastinum and esophagus. Hum Pathol 2004; 35: 1288-91.

4. de Montpréville VT, Mussot S, Gharbi N, Dartevelle P, Dulmet E. Paraganglioma with ganglioneuromatous component located in the posterior mediastinum. Ann Diagn Pathol 2005; 9: 110-4.

5. Weissferdt A, Kalhor N, Liu H, et al. Thymic neuroendocrine tumors (paraganglioma and carcinoid tumors): a comparative immunohistochemical study of 46 cases. Hum Pathol 2014; 45: 2463-70.

6. Okubo Y, Nemoto T, Wakayama M, et al. Gangliocytic paraganglioma: a multi-institutional retrospective study in Japan. BMC Cancer 2015; 15: 269.

7. Ogata S, Horio T, Sugiura Y, Aiko S, Aida S. Duodenal gangliocytic paraganglioma with regional lymph node metastasis and a glandular component. Pathol Int 2011; 61: 104-7. 